\title{
Sementes de Zanthoxylum rhoifolium Lam.: tolerância à secagem, ao armazenamento e ao descongelamento
}

\author{
Bruno Jan Schramm Corrêa $\odot^{1 *}$ Luciana Magda de Oliveira $0^{1}$ Alexandra Cristina Schatz Sá임 \\ Vanessa Giseli Dambros ${ }^{1}$ Laís da Rosa Delfes ${ }^{1}$ Betel Cavalcante Lopes ${ }^{1}{ }^{1}$ Anieli Cioato de \\ Souza ${ }^{1}$
}

${ }^{1}$ Universidadedo Estado de Santa Catarina, Av. Luiz de Camões, 2090 - Conta Dinheiro, CEP 88520-000, Lages - SC, Brasil

Technical note
*Corresponding author:
brschramm74@gmail.com

Palavras-chave:

Sementes ortodoxas

Qualidade Fisiológica

Dessecação

Keywords:

Ortodox Seeds

Physiological Quality

Dissecation

Received in

2020/07/20

Accepted on

2020/12/22

Published in

2021/01/12

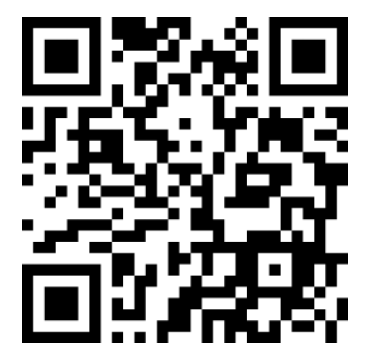

DOI: http://dx.doi.org/ 10.34062/afs.v7i4.10854

\section{(cc) BY}

RESUMO: O armazenamento apropriado de sementes florestais é um importante quesito para preservar a sua qualidade fisiológica. Desta forma, objetivou-se realizar a classificação fisiológica de sementes de Zanthoxylum rhoifolium de acordo com sua tolerância à dessecação e ao armazenamento e avaliar diferentes tratamentos para descongelamento. As sementes foram expostas à dessecação até $5 \%$ de teor de água e armazenadas em $-20^{\circ} \mathrm{C}$ durante três meses. Em seguida, as sementes foram submetidas aos seguintes tratamentos para descongelamento: câmara a $25{ }^{\circ} \mathrm{C}$, estufa a $40{ }^{\circ} \mathrm{C}$, banho-maria a $50{ }^{\circ} \mathrm{C}$, micro-ondas e em temperatura ambiente de 18 a $25^{\circ} \mathrm{C}$. A viabilidade das sementes foi avaliada pelo teste de tetrazólio em todas as etapas. As sementes de Z. rhoifolium são tolerantes à dessecação até $5 \%$ de umidade e ao armazenamento em $-20^{\circ} \mathrm{C}$, sendo classificadas como ortodoxas. As sementes podem ser descongeladas em câmara $\left(25^{\circ} \mathrm{C}\right)$, estufa $\left(40^{\circ} \mathrm{C}\right)$ e em temperatura ambiente $\left(18\right.$ a $\left.25^{\circ} \mathrm{C}\right)$, porém, para manter a viabilidade, indica-se o descongelamento a $25^{\circ} \mathrm{C}$ constantes.

\section{Seeds of Zanthoxylum rhoifolium Lam.: tolerance to drying, storage, and thawing}

\begin{abstract}
Proper storage of forest seeds is an important requirement to preserve their physiological quality. The objective of this study was to perform the physiological classification of Zanthoxylum rhoifolium seeds according to their tolerance to desiccation and storage and evaluate different treatments of thawing. The seeds were exposed to desiccation up to $5 \%$ water content and stored at -20 ${ }^{\circ} \mathrm{C}$ for three months. Then, the seeds were subjected to the following treatments for thawing: chamber at $25^{\circ} \mathrm{C}$, oven at $40^{\circ} \mathrm{C}$, water bath at $50{ }^{\circ} \mathrm{C}$, microwave and at room temperature from 18 to $25^{\circ} \mathrm{C}$. The viability of the seeds was evaluated by the tetrazolium test in all stages. The seeds of Z. rhoifolium are tolerant to desiccation up to $5 \%$ water content and to storage at $-20^{\circ} \mathrm{C}$, being classified as orthodox. The seeds can be thawed in chamber $\left(25^{\circ} \mathrm{C}\right.$.), oven $\left(40^{\circ} \mathrm{C}\right)$ and at room temperature $\left(18\right.$ to $\left.25^{\circ} \mathrm{C}\right)$, however, to maintain viability, it is recommended the thawing at constant $25^{\circ} \mathrm{C}$.
\end{abstract}




\section{Côrrea et al.}

\section{Introdução}

$\mathrm{O}$ armazenamento adequado consiste como um importante requisito para preservar a qualidade de sementes. Caracterizar o desempenho fisiológico de sementes florestais permite estimar a viabilidade das mesmas, auxiliando na tomada de decisões quanto ao plantio, produção de mudas, conservação genética, manutenção de germoplasma e nas pesquisas na área de tecnologia e fisiologia de sementes (Gasparin et al. 2018).

A classificação de sementes, segundo seu potencial fisiológico durante a secagem e o armazenamento pode ser dividida em três categorias: ortodoxas, intermediárias e recalcitrantes. Sementes ortodoxas suportam baixos níveis de teor de água e podem ser conservadas em baixas temperaturas. Sementes intermediárias suportam a dessecação a baixos níveis de água (10 a 12\%), mas perdem viabilidade quando armazenadas em baixas temperaturas, já as sementes recalcitrantes perdem a viabilidade quando expostas à dessecação e ao armazenamento em temperaturas reduzidas (Hong e Ellis 1996; Sacandé et al. 2004).

Além da classificação das sementes quanto ao desempenho fisiológico e armazenamento, informações sobre o descongelamento adequado das sementes são importantes na manutenção da viabilidade do material quando exposto a temperaturas abaixo de zero (Salomão 2002; Fonseca et al. 2012). Por isso, são necessárias metodologias adequadas para cada espécie, visto que cada uma responde de forma distinta ao congelamento e ao descongelamento (Saleh et al. 2017).

Zanthoxylum rhoifolium Lam. (Rutaceae) é uma árvore decídua de ocorrência em diversas formações fitogeográficas brasileiras, sendo caracterizada pelo tronco aculeado e médio porte, com o nome popular de mamica-de-cadela ou juvevê. A madeira desta espécie é utilizada na confecção de cabos de ferramentas e construção civil, apresenta potencial para a restauração de áreas degradadas, paisagismo e, principalmente, potencial medicinal devido às suas propriedades tônicas, estomáquicas, antimicrobianas e antiparasitárias (Carvalho 2003; Krause et al. 2013). Ainda que apresente variados usos e potencialidades, $Z$. rhoifolioum é uma espécie que possui poucos estudos relacionados a fisiologia de suas sementes, somente citações sobre seu desempenho fisiológico em armazenamento (Carvalho 2003; Salomão et al. 2005).

Objetivou-se realizar a classificação fisiológica de sementes de Z. rhoifolium de acordo com a tolerância à dessecação e ao armazenamento, e avaliar métodos de descongelamento.

\section{Material e métodos}

As sementes de Z. rhoifolium foram coletadas no município de Dois Vizinhos, Paraná, em área natural de coleta. A altitude média do local é de 409 metros, com clima do tipo 'Cfa' subtropical úmido (Alvares et al. 2018). A coleta ocorreu no mês de fevereiro de 2018, diretamente de três árvores, com auxílio de podão. Após a coleta, as sementes foram secas na sombra, em temperatura ambiente, por $48 \mathrm{~h}$ e submetidas ao beneficiamento manual, com auxílio de peneira (Carvalho, 2003).

A classificação fisiológica de sementes de $Z$. rhoifolium em armazenamento foi realizada seguindo o protocolo proposto por Hong e Ellis (1996) e adaptado por Sacandé et al. (2004) (Figura 1). Foi seguido o protocolo a partir da etapa de secagem das sementes a $5 \%$ de umidade, visto que essas foram colhidas com $9 \%$.

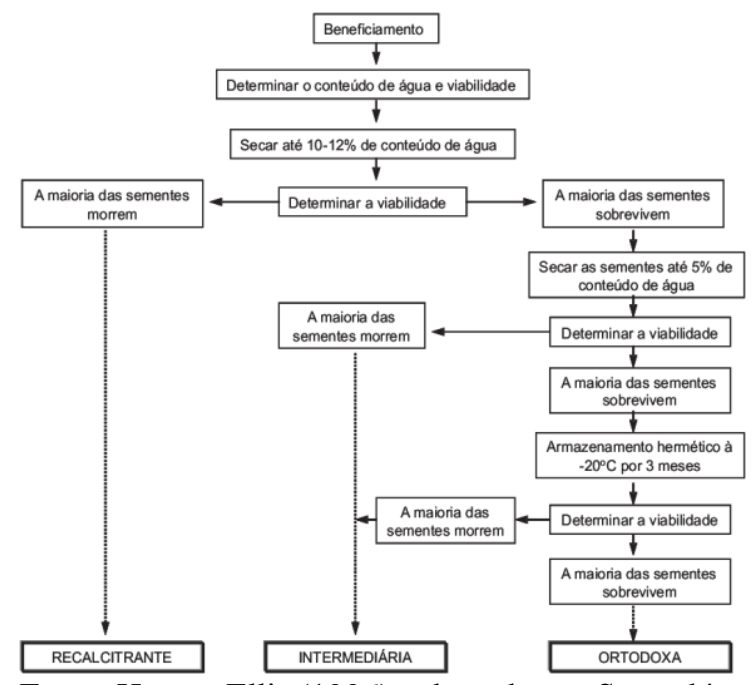

Fonte: Hong e Ellis (1996), adaptado por Sacandé et al. (2004).

Figura 1. Protocolo para classificação fisiológica de sementes de acordo com a tolerância à dessecação e ao armazenamento.

Foi realizado o método de secagem rápida, por meio do acondicionamento das sementes em recipientes herméticos, expostas à sílica gel, em temperatura de $25{ }^{\circ} \mathrm{C}$, até a obtenção de aproximadamente $5 \%$ de umidade. A massa foi verificada por meio de pesagem em balança analítica a cada $1 \mathrm{~h}$ durante as primeiras $12 \mathrm{~h}$ e, a partir deste ponto após $12 \mathrm{~h}$, totalizando $24 \mathrm{~h}$. A perda de água das sementes foi monitorada por meio do cálculo de diferença de massa.

$M=\frac{(100-C A i)}{(100-C A d)} \times M i$

M é a massa (g) no conteúdo de água desejado, Mi é a massa (g) no conteúdo de água inicial, CAi é o conteúdo de água inicial (\% base 
úmida) e CAd é o conteúdo de água desejado (\% base úmida)

As sementes com teor de água de 5\% foram acondicionadas em recipiente hermético e armazenadas em freezer regulado a $-20^{\circ} \mathrm{C}$, durante três meses. Após este período, as sementes foram expostas a diferentes tratamentos para descongelamento, onde: $\mathrm{T} 1$ - descongelamento em temperatura ambiente $\left(18\right.$ a $\left.25{ }^{\circ} \mathrm{C}\right)$ durante $24 \mathrm{~h} ; \mathrm{T} 2$ - banho-maria a $50^{\circ} \mathrm{C}$ durante $1 \mathrm{~h}$; $\mathrm{T} 3$ - micro-ondas durante 1,5 min; T4 - câmara de germinação (tipo Biochemical oxygen demand (B.O.D.)) a $25{ }^{\circ} \mathrm{C}$ durante $24 \mathrm{~h}$; T5 - estufa a $40{ }^{\circ} \mathrm{C}$ durante $4 \mathrm{~h}$ (Fonseca et al. 2012; Alencar et al. 2018).

Em todas as etapas e após os diferentes tratamentos de descongelamento, determinou-se a viabilidade das sementes pelo teste de tetrazólio. As sementes foram seccionadas lateralmente $\mathrm{e}$ embebidas em solução de tetrazólio em concentração de $0,05 \%$ durante $48 \mathrm{~h}$, em B.O.D. a $25{ }^{\circ} \mathrm{C}$, na ausência de luz. Neste caso, foi seguida metodologia determinada por testes preliminares (dados não apresentados) com diferentes lotes da espécie. Ressalta-se que não foi realizado teste de germinação devido à dormência das sementes e a falta de metodologia adequada para sua superação.

Foram utilizadas quatro repetições de 25 sementes por tratamento, seguindo um delineamento inteiramente casualizado. Foi testada a normalidade e homogeneidade dos dados obtidos dos tratamentos para descongelamento. Atendendo aos pressupostos de normalidade, os dados foram submetidos à análise de variância e as médias comparadas pelo teste t para ao nível de 5\% de probabilidade com auxílio do programa estatístico Sisvar 5.6.

\section{Resultados}

As sementes de Z. rhoifolium apresentaram teor de água inicial e viabilidade de $9 \%$ e $74 \%$, respectivamente. Após a secagem em sílica gel, as sementes apresentaram 5\% de umidade e $80 \%$ de viabilidade pelo teste de tetrazólio. Além da tolerância à secagem até este nível de umidade, as sementes de $Z$. rhoifolium não tiveram a viabilidade significativamente reduzida (64\%) quando foram expostas à temperatura de $-20{ }^{\circ} \mathrm{C}$ durante $\mathrm{o}$ armazenamento. Não foi verificada diferença significativa entre as etapas.

De acordo com os resultados obtidos após o descongelamento, as sementes que mantiveram a viabilidade foram as descongeladas em B.O.D. a 25 ${ }^{\circ} \mathrm{C}$ (T4), seguido pelo descongelamento em estufa a $40{ }^{\circ} \mathrm{C}$ (T5), temperatura ambiente $\left(18\right.$ a $\left.25^{\circ} \mathrm{C}\right)(\mathrm{T} 1)$, banho-maria (T2) e em menor número em microondas (T3), com diferenças estatísticas significativas entre os tratamentos (Tabela 1).

Tabela 1. Viabilidade segundo teste de tetrazólio (TZ) de sementes de Zanthoxylum rhoifolium em diferentes tipos de descongelamento.

\begin{tabular}{cc}
\hline Tratamentos & Médias* \\
\hline T1 - Temp. ambiente & $48 \mathrm{ab}$ \\
T2 - Banho-maria & $45 \mathrm{~b}$ \\
T3 - Micro-ondas & $8 \mathrm{c}$ \\
T4 - B.O.D. & $64 \mathrm{a}$ \\
T5 - Estufa & $58 \mathrm{ab}$ \\
\hline C.V.** & 30,7 \\
\hline
\end{tabular}

* Médias seguidas por uma mesma letra na coluna, não diferem estatisticamente ( $t$ de Student, $p<0,05$ ) $* *$ C.V. $=$ coeficiente de variação.

\section{Discussão}

Sementes com desempenho ortodoxo são caracterizadas por tolerarem níveis reduzidos de umidade e armazenamento em baixas temperaturas (Bewley et al. 2013). A capacidade de sementes de Z. rhoifolium em apresentarem viabilidade após secagem até 5\% de umidade é um fator indicativo de espécie ortodoxa (Hong e Ellis 1996; Saleh et al. 2017). Com este desempenho, reafirma-se a hipótese de que esta espécie possui sementes ortodoxas (Carvalho 2003; Salomão et al. 2005). Estes resultados convergem com as observações de Tweddle et al. (2003) para a família Rutaceae, que apresentam majoritariamente espécies com sementes ortodoxas. Aliado a isso, pesquisas recentes sobre o desempenho durante secagem e armazenamento de sementes florestais tropicais, tais como Casearia sylvestris Swartz (Salicaceae) (Nery et al. 2014), Miconia albicans (Sw.) (Melastomaceae), Styrax camporum Pohl. (Styracaceae), Piptadenia gonoacantha (Mart.) J. F. Macbr (Mimosoideae) (Mayrinck et al. 2016) e Attalea speciosa Mart. ex Spreng (Arecaceae) (Saleh et al. 2017) demonstraram um predomínio de sementes ortodoxas.

$\mathrm{O}$ descongelamento rápido em micro-ondas foi o tratamento que prejudicou a viabilidade em relação aos demais tratamentos, seguido por banhomaria. Tais resultados conduzem a hipótese de que o descongelamento rápido pode danificar seus tecidos internos e externos (Bewley 2013; Zhang et al. 2014). De forma semelhante, as sementes de Apuleia leiocarpa (Vogel) J. F. Macbr. (Fabaceae) (Salomão et al. 2002), Jatropha curcas L. (Euphorbiaceae) (Goldfarb et al. 2010) e Handroanthus spongiosus (Rizzini) S. Grose (Bignoniaceae) (Alencar et al. 2018) apresentaram maior qualidade fisiológica em descongelamento lento quando comparado ao descongelamento rápido.

Ressalta-se que podem ser utilizados três métodos para o descongelamento (B.O.D., estufa e temperatura ambiente), entretanto, indica-se o uso de B.O.D. a $25^{\circ} \mathrm{C}$ como método mais adequado para o descongelamento de sementes desta espécie, pois mantém constantes a temperatura e condições de descongelamento. 
É valido salientar a importância de bioensaios que elucidem os métodos adequados de armazenamento e descongelamento de sementes florestais (Fonseca et al. 2012; Menegatti et al. 2017). Sem a obtenção dos valores de viabilidade em diferentes formas de descongelamento, a classificação fisiológica da espécie poderia ser realizada de forma errônea.

\section{Conclusões}

As sementes de Zanthoxylum rhoifolium são tolerantes à dessecação até $5 \%$ de umidade e ao armazenamento em $-20^{\circ} \mathrm{C}$, sendo classificadas como ortodoxas.

As sementes de Zanthoxylum rhoifolium podem ser descongeladas em câmara B.O.D a $25^{\circ} \mathrm{C}$, estufa a $40{ }^{\circ} \mathrm{C}$ e temperatura ambiente $\left(18\right.$ a $\left.25^{\circ} \mathrm{C}\right)$, entretanto, recomenda-se o descongelamento a $25^{\circ} \mathrm{C}$ constantes para melhor preservação de viabilidade.

\section{Agradecimentos}

Os autores agradecem a Coordenação de Aperfeiçoamento de Pessoal de Nível Superior (CAPES) e ao Conselho Nacional de Desenvolvimento Científico e Tecnológico (CNPq) pelo auxílio financeiro durante a realização da pesquisa.

\section{Referências}

Alencar SS, Freire JNT, Gomes RA, Silva JJ, Araújo MN, Dantas BF (2018) Descongelamento de sementes crioconservadas de Handroanthus spongiosus (Rizzini) S. Grose. Informativo Abrates, 28(1): 51-54. Disponível em: https://ainfo.cnptia.embrapa.br/digital/bitstream/ite m/197387/1/Barbara-2.pdf. Acesso em: 02 jul. 2020.

Alvares CA, Sentelhas PC, Stape JL (2018) Modeling monthly meteorological and agronomic frost days, based on minimum air temperature, in Center-Southern Brazil. Theoretical and Applied Climatology, 134. doi: 10.1007/s00704-017-2267-6

Bewley JD, Bradford KJ, Hilhorst HWM, Nonogaki H. (2012) Seeds: physiology of development, germination, and dormancy. $3^{\text {th }}$ Edition. Nova York: Springer. $407 \mathrm{p}$.

Carvalho PER (2003) Espécies florestais brasileiras: recomendações silviculturais, potencialidades e uso da madeira. Colombo: EMBRAPA/CNPAF. 640 p.

Fonseca AG, Matuda JJ, Almeida JO, Nunes UR, Machado EL (2012) Qualidade fisiológica de sementes de Pinus elliotti Engelm. submetidas a diferentes métodos de armazenamento. Cerne, 18(3): 457-463. doi: 10.1590/S010477602012000300013
Gasparin E, Araujo MM, Franco EH, Oliveira LM (2018) Armazenamento de sementes de espécies florestais. In: Araujo MM, Navroski MC, Schorn LA (ed) Produção de Sementes e Mudas - um enfoque à Silvicultura. Santa Maria: UFSM, 99-120.

Goldfarb M, Duarte MEM, Mata MERMC (2010) Armazenamento criogênico de sementes de pinhãomanso (Jatropha curcas L.) Euphorbiaceae. Revista Biotemas, 23(1): 27-33. doi: 10.5007/21757925.2010v23n1p27

Hong TD, Ellis RH (1996) A protocol to determine seed storage behaviour. Rome: International Plant Genetic Resources Institute. 55 p.

Krause MS, Bonetti AF, Turnes JM, Dias JFG, Miguel OG, Duarte MR (2013) Fitoquímica e atividades biológicas de Zanthoxylum rhoifolium Lam., Rutaceae - mini revisão. Visão Acadêmica, 14: 118-127. doi: 10.5380/acd.v14i4.33992.

Mayrinck RC, Vaz TA, Davide AC (2016) Physiological classification of forest seeds regarding the desiccation tolerance and storage behavior. Cerne, 22 (1): 85 - 92. doi: $10.1590 / 01047760201622012064$.

Menegatti R, Mantovani A, Navroski MC, Guollo K, Vargas OF, Souza AG (2017) Germinação de sementes de Mimosa scabrella Benth. submetidas a diferentes condições de temperatura, armazenamento e tratamentos pré-germinativos. Revista de Ciências Agrárias, 40(2): 1-10. doi: 10.19084/RCA16153

Nery MC, Davide AC, Silva EAA Da, Soares GCM, Nery FC (2014) Classificação fisiológica de sementes florestais quanto a tolerância à dessecação e ao armazenamento. Cerne, 20(3): 477-483. DOI: $10.1590 / 01047760201420031450$.

Sacandé M, Joker D, Dulloo ME, Thompsen KA (2004) Comparative storage biology of tropical tree seeds. Rome: International Plant Genetic Resources Institute. 363 p.

Saleh EOL, Luis ZG, Scherwinski-Pereira JE (2017) Determination of physiological and environmental conditions for the storage of babassu palm seeds (Attalea speciosa). Seed Science and Technology, 45(1): 139-150. doi: 10.15258/sst.2017.45.1.24.

Salomão AN (2002). Respostas de sementes de espécies tropicais a exposição ao nitrogênio líquido. Brazilian Journal of Plant Physiology, 14(2): 133 138. doi: 10.1590/S1677-04202002000200008. 


\section{Côrrea et al.}

Salomão AN, Santos IRI, Mundim RC (2002) Estabelecimento de método para congelamento e descongelamento de sementes de Apuleia Leiocarpa (Vog.) Macbr. (Caesalpinaceae). Brasília: Embrapa Recursos Genéticos e Biotecnologia (Circular Técnica, 19).

Salomão NA, Walter BMT, Cavalcanti TB, Medeiros MB de, Santos IRI dos, Santos AA, Santos GP dos, Mundim RC, Pereira JB, Rezende JM, Moreira GA (2005) Desenvolvimento de metodologias para a conservação de germoplasma semente resgatado em áreas de aproveitamento de cinco hidrelétricas no Bioma Cerrado. Brasília: Embrapa Recursos Genéticos e Biotecnologia. 28 p.

Tweddle JC, Turner RM, Dickie JB (2003) Seed Information Database. Disponível em: <http:// www.rbgkew.org.uk/data/sid>. Acesso em: 20 jul. 2020.

Zhang N, Wen B, Ji M, Yan Q (2014) Lowtemperature storage and cryopreservation of grapefruit (Citrus paradisi Macfad.) seeds. Cryoletters, 35(5): 418-426. 